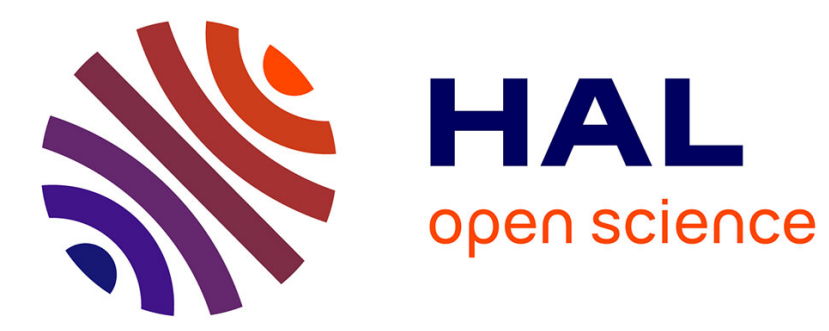

\title{
Les paradoxes de la Region en Allemagne, entre réseaux et territoires : la région, outil de déterritorialisation ?
}

Christophe Queva

\section{To cite this version:}

Christophe Queva. Les paradoxes de la Region en Allemagne, entre réseaux et territoires : la région, outil de déterritorialisation ?. Annales de géographie, 2007, 653 (1), p. 41-64. 10.3917/ag.653.0041 . halshs-01708874

\section{HAL Id: halshs-01708874 \\ https://shs.hal.science/halshs-01708874}

Submitted on 14 Feb 2018

HAL is a multi-disciplinary open access archive for the deposit and dissemination of scientific research documents, whether they are published or not. The documents may come from teaching and research institutions in France or abroad, or from public or private research centers.
L'archive ouverte pluridisciplinaire HAL, est destinée au dépôt et à la diffusion de documents scientifiques de niveau recherche, publiés ou non, émanant des établissements d'enseignement et de recherche français ou étrangers, des laboratoires publics ou privés. 


\section{LES PARADOXES DE LA REGION EN ALLEMAGNE, ENTRE RÉSEAUX ET TERRITOIRES : LA RÉGION, OUTIL DE DÉTERRITORIALISATION ?}

\section{Christophe Queva}

Armand Colin | «Annales de géographie »

2007/1 n 653 | pages 41 à 64

ISSN 0003-4010

ISBN 9782200923129

Article disponible en ligne à l'adresse :

https://www.cairn.info/revue-annales-de-geographie-2007-1-page-41.htm

\section{Pour citer cet article :}

Christophe Queva, « Les paradoxes de la Region en Allemagne, entre réseaux et territoires : la région, outil de déterritorialisation ? », Annales de géographie 2007/1 ( $\left.\mathrm{n}^{\circ} 653\right)$, p. 41-64.

DOI 10.3917/ag.653.0041

Distribution électronique Cairn.info pour Armand Colin.

(C) Armand Colin. Tous droits réservés pour tous pays.

La reproduction ou représentation de cet article, notamment par photocopie, n'est autorisée que dans les limites des conditions générales d'utilisation du site ou, le cas échéant, des conditions générales de la licence souscrite par votre établissement. Toute autre reproduction ou représentation, en tout ou partie, sous quelque forme et de quelque manière que ce soit, est interdite sauf accord préalable et écrit de l'éditeur, en dehors des cas prévus par la législation en vigueur en France. Il est précisé que son stockage dans une base de données est également interdit. 


\title{
Les paradoxes de la Region en Allemagne, entre réseaux et territoires : la région, outil de déterritorialisation?
}

\author{
At the crossroad between territory and network: \\ the paradoxical notion of Region in Germany. \\ Is Region a deterritorializating device
}

Christophe Queva

Département de Géographie, Université Michel de Montaigne (Bordeaux III)

Résumé Depuis la fin des années 1990, la région (Region) est devenue, en Allemagne, un outil central d'aménagement " par le bas » et de développement (Raumentwicklung), par opposition à la traditionnelle planification spatiale (Raumplanung). Mais la Region reste une entité ambiguë, entre territoires et réseaux, structures formelles et informelles. Il s'agit en fait de distinguer deux types de régions : la région d'aménagement ou de planification, et la région au sens large. Les deux premières correspondent à un même espace de planification exogène, considéré soit du point de vue du Bund (Raumordnungsregion), soit de celui des Länder (Planungsregion). La région au sens large (Region) désigne, quant à elle, un espace d'action local fondé sur des coopérations souvent réticulaires et informelles. Or, ce sont les relations entre ces deux entités qui font, en Allemagne, le développement régional (Regionalentwicklung) - on parlerait en français de " développement local ». Il se dessine ainsi une dynamique paradoxale, dans laquelle domine dans un premier temps le rejet de toute rigidité territoriale, par le choix du flou dans des coopérations thématiques et réticulaires. Par la suite, se manifeste la nécessité d'un retour au territoire, plus ou moins assumé et revendiqué par les acteurs locaux. Des exemples nationaux et régionaux (région d'aménagement de Thuringe du Sud Ouest) viennent illustrer cette réflexion.

Abstract From the end of the 1990s onwards, German spatial planning experiences have been enhancing the value of the notion of Region as a key tool in "bottom-up spatial planning"; thus moving on from Raumplanung (spatial planning) to Raumentwicklung (spatial development). However, the region remains ambivalent, standing as it is between territories and networks, formal and informal structures. There are two identifiable types of regions in Germany : the region as defined by spatial planning and the region in general. The first one (Raumordnungsregion when applied to the Bund, and Planungsregion when applied to the Länder) is an external planning instrument, thus coordinating local actors and their projects. This is grounded upon the territorial divisions within each Land. Regions in general (Region) are spaces intended for local action, founded upon reticular patterns of cooperation rather than upon territorial ones, such as urban networks, regional development concepts, etc. But local/regional development rests upon these two interweaving/interacting entities. The play on scales and the ambivalent relations between networks and territories in regional development are at the core of this article. Eventually, this leads us to a paradoxical form of dynamism. On the one hand, the dismissal of territorial rigidity and the choice of weak, thematic and reticular cooperation seem to be 
prevailing. But on the other hand, the need to go back to the notion of territory is still felt, if not always accepted, by local actors. The region would first be used as a deterritorializating device at the stage of regional development projects, before being turned into a reterritorializating tool afterwards, when implementing actions and projects. I will use a few national (German) and regional examples (the spatial planning region of South-West Thuringe) to illustrate these ideas.

Mots-clés Région, acteurs, aménagement, développement local, territoires, réseaux.

Key-words Region, actors, spatial planning, local/regional development, territories, networks.

Dans le contexte européen, l'échelon régional est devenu un niveau d'organisation et d'action de plus en plus valorisé par les aménageurs et les politiques. C'est notamment le cas en Allemagne, où la région constitue, depuis les années 1980, non plus seulement l'échelon d'application des politiques d'aménagement des Länder, mais aussi celui de la mobilisation des acteurs locaux, dans le cadre des régions dites d'aménagement (Raumordnungsregionen) ou de planification (Planungsregionen). Les deux termes se rapportent en fait à la même entité, considérée soit du point de vue du gouvernement fédéral (le Bund) pour le premier, soit de celui des gouvernements fédérés (les Länder) pour le second. Le découpage en 97 régions d'aménagement ${ }^{1}$ est clairement établi et leurs compétences sont bien définies: sans être une collectivité territoriale, la région d'aménagement est un outil de nature à la fois exogène et endogène. Ainsi, chaque Land élabore des schémas d'aménagement planifié de son territoire, dont la mise en œuvre revient aux régions d'aménagement qui le composent. Par ailleurs, depuis la réforme de la loi sur l'aménagement (1998), l'échelon régional est associé au développement local (Regionalentwicklung), aux projets élaborés par les acteurs locaux, ou encore à un nouveau type d'action : la gouvernance régionale.

Ces caractéristiques sont toutefois synonymes d'un élargissement réel de la notion de région à des champs autres que ceux de la stricte région d'aménagement. Ainsi, dans les textes administratifs du bureau fédéral chargé de l'aménagement de l'espace $\left(B B R^{2}\right)$ comme dans le discours des acteurs régionaux, la région comporte une multitude de références qui en font une entité particulièrement floue, selon qu'on la considère dans son sens le plus strict (région d'aménagement) ou au sens large (Region). Elle peut paradoxalement présenter un fort degré de territorialisation - espace de planification et d'aménagement - ou apparaître quasiment déterritorialisée en étant synonyme de réseaux informels, de coopérations d'acteurs, sans fondements spatiaux clairement identifiés. Ce relatif détachement vis-à-vis de l'espace expliquerait ainsi le

1 Le nombre de régions d'aménagement est officiellement de 97 (données du Bundesamt für Bauwesen und Raumordnung). Certains Länder peuvent toutefois subdiviser ces régions en plusieurs sous-régions de planification, ce qui fait que l'on peut trouver parfois le chiffre de 115 régions d'aménagement/de planification au total.

2 Bundesamt für Bauwesen und Raumordnung (BBR). 
privilège accordé, non pas aux territoires (Territorien ou Gebiete), comme en France avec les Pays (LOADDT ${ }^{3}$, 1999) par exemple, mais aux réseaux (Netzwerke), pour constituer les projets d'aménagement et de développement local et régional : "Planen und Handeln in "Netzen" wird wichtiger, insbesondere auf der regionalen Ebene» $(B B R, 2000)^{4}$.

Cet article vise à interroger la territorialité des expériences régionales en Allemagne : en quoi l'espace régional peut-il apparaître plus réticulaire que territorial ? Ces deux notions ne sont-elles pas plutôt complémentaires ? Le rejet apparent du territoire dans les expériences de développement régional est-il de l'ordre de l'utopie, appelant ensuite une nécessaire territorialisation des projets régionaux ? Les résultats de recherches bibliographiques sur la question à l'échelle de l'Allemagne, et ceux d'enquêtes effectuées dans la région d'aménagement de Thuringe du Sud-Ouest tentent d'apporter quelques réponses à ces questions, avec l'hypothèse que la déterritorialisation $a$ priori, associée à la Region, appelle en fait un retour au territorial a posteriori, qui est plus ou moins assumé et revendiqué dans les actuelles expériences régionales d'aménagement.

\section{Un outil régional volontairement flou et pluriel}

Véritable outil récemment revalorisé de l'aménagement en Allemagne, la « région d'aménagement » ou « de planification »se définit à la fois comme un objet d'aménagement en tant qu'échelon-relais des politiques de planification spatiale du Land, et comme un cadre pour l'aménagement, en tant que structure de coordination de projets locaux. Elle reste un outil d'aménagement du haut vers le bas, tout en étant un espace fonctionnel, appuyé sur un réseau hiérarchisé de villes centrales ${ }^{5}$. Sur un plan territorial, les régions d'aménagement se fondent sur les découpages déjà existants, à l'intérieur des limites de chaque Land et regroupent plusieurs Kreise (arrondissements) - circonscriptions administratives locales situées audessus des communes. La figure 1 permet de situer les 97 régions d'aménagement allemandes et, en particulier notre terrain d'étude : la région de Thuringe du Sud-Ouest.

Le nom de beaucoup de ces régions d'aménagement/de planification illustre bien l'idée d'un découpage exogène (Mecklenbourg de l'Ouest, Hesse orientale, Thuringe du Sud-Ouest, etc.) : elles sont avant tout un espace de planification, un découpage effectué par les Länder.

Mais on peut parler aujourd'hui d'une réelle complexification, liée au fait qu'au sein de ces régions d'aménagement (par le haut), les projets de développement établis par les acteurs locaux (concepts de développement

3 Loi d'orientation pour l'aménagement et le développement durable du territoire.

4 Ce sont les réseaux qui, tant au niveau de la planification que de l'action, se retrouvent au premier plan, en particulier au niveau régional.

5 L'idée de la région d'aménagement comme espace fonctionnel, voire identitaire, est développée plus loin. 


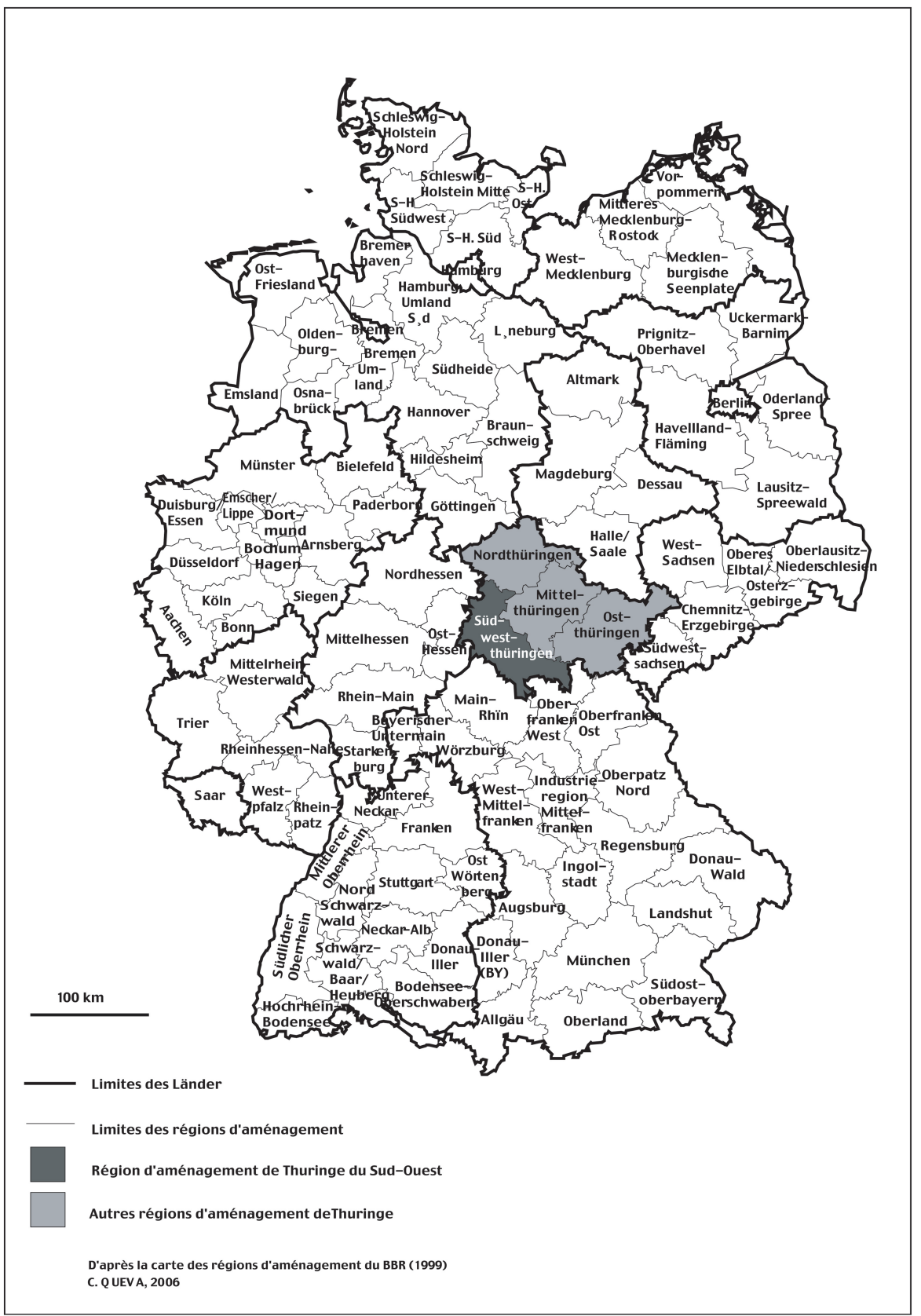

Fig. 1 Les régions d'aménagement en Allemagne (localisation de la région de Thuringe du Sud-Ouest). Planning regions in Germany (including South-West Thuringe). 
régional, conférences régionales, réseaux de villes régionaux, etc.) en viennent également à être considérés comme régionaux. Or, ils correspondent à des projets de développement "par le bas » souvent intercommunal, rarement à l'échelle de la région d'aménagement. La notion de Region désigne ainsi aujourd'hui une grande variété d'entités - locales ou supra-locales dont les acteurs peuvent paraître difficiles à identifier. La Region est un échelon de plus en plus flou, alors qu'elle constitue un enjeu réel en termes de développement local - enjeu d'autant plus marqué que les interventions européennes reposent désormais sur une vision du développement valorisant l'initiative endogène, autour d'espaces de projet et de coopération entre acteurs locaux ${ }^{6}$.

\subsection{Une régionalisation croissante de l'aménagement et du développement}

Historiquement, on peut parler d'un mouvement de balancier entre valorisation et mise à l'écart de la région comme outil d'aménagement. Les premières expériences d'aménagement en Allemagne se sont mises en place à partir d'initiatives régionales (Guder, 2003): syndicat de planification autour de Berlin et des communes voisines (Zweckverband Gross-Berlin, 1916), syndicat d'agglomération du district minier de la Ruhr (Siedlungsverband Rubrkoblenbezirk, 1920). Ces expériences correspondent à des coopérations volontaires dans une optique de planification spatiale "régionale », mais dans le cas particulier d'espaces urbanisés connaissant une industrialisation marquée. Par la suite, le poids croissant de l'État central - sous le régime national-socialiste, puis en RDA - ou celui des Länder en RFA, ont évacué les initiatives locales ou régionales ${ }^{7}$. Ainsi, après 1945 et jusqu'à la fin des années 1990, la région n'a plus joué de rôle majeur dans l'aménagement de l'ex-RFA, et encore moins dans celui de l'ex-RDA où la Territorialplanung (planification territoriale) correspondait à un aménagement centralisé du territoire national : l'échelon infra-étatique des Bezirke n'était alors qu'un échelon-relais pour la mise en œuvre de la planification décidée par l'État central. En RFA, le système traditionnel d'aménagement était et reste aujourd'hui encore planifié et hiérarchisé, et il accorde un rôle majeur aux Länder. Ainsi, le Bund élabore une planification générale de l'espace national (Raumplanung) qui est plus indicative que directive. La tâche de l'aménagement, dans la logique du modèle fédéral, revient avant tout aux Länder. Cette planification du Land (Landesplanung) correspond à des orientations générales, divisées en sous branches régionales et locales. À partir de leur création en 1981, les régions de planification - nommées régions d'aménagement par le Bund - mettent en place une planification régionale (Regionalplanung) en lien avec les indications du Land. Enfin,

6 Je pense ici notamment aux politiques régionales encourageant la coopération intercommunale (programmes LEADER+, INTERREG, etc.)

7 Les Länder sont des États (fédérés dans un Etat fédéral, le Bund), et non des régions. 
les collectivités locales (communes, Kreise) sont chargées de la mise en œuvre concrète de l'aménagement dans le cadre d'une planification locale, la Bauleitplanung, en lien avec les indications du Land et de la région d'aménagement. Le schéma d'organisation général repose ainsi sur un système de relations à double sens entre les différents échelons territoriaux, le Bund et les Länder émettant des planifications générales à petite échelle, et les collectivités locales soumettant elles-mêmes des planifications à grande échelle, en lien avec les planifications des échelons supérieurs. Dans l'ensemble, c'est l'échelon étatique du Land qui reste central. Ce schéma régit encore l'aménagement dans sa version traditionnelle (Planung), dans l'ensemble de l'Allemagne réunifiée.

Or, avec la Réunification et l'apparition de réelles disparités régionales, de nouveaux instruments d'aménagement ont été créés dans le cadre d'un développement spatial (Raumentwicklung), mis en place "par le bas » afin de mieux prendre en compte les attentes et les projets des acteurs locaux. C'est alors qu'un échelon régional - beaucoup plus proche du local - se voit revalorisé, au point que certains parlent d'une véritable «inflation de coopération régionale »(Diller, 2002). Du point de vue des politiques d'aménagement en tant que telles se manifeste en effet, depuis la fin des années 1990, le souci de se détacher de la traditionnelle planification spatiale (Raumplanung), jugée trop centralisée - à l'échelon du Bund et surtout des Länder - et hiérarchisée, au profit de l'idée de développement spatial (Raumentwicklung), que l'on souhaite plus informel, thématique et endogène, dans un cadre qualifié de régional (Regionalentwicklung). On retrouve d'ailleurs cette idée en France avec le passage de l'aménagement $d u$ territoire, centralisé et hiérarchisé, au développement local - associant, selon la formule de la DATAR, " un territoire, un projet, un contrat ", et se détachant ainsi progressivement du modèle gaullien d'organisation territoriale (Behar, 2000). En France comme en Allemagne, ces mutations s'expliquent essentiellement par la nécessité, pour les acteurs locaux, d'agir à un niveau supra-communal pour plus d'efficacité et, en Allemagne, le développement régional est présenté comme un contrepoids possible aux égoïsmes locaux et aux pouvoirs étatiques. Il s'agit dès lors de tenter de cerner ce nouvel outil de développement qu'est la Region en Allemagne.

\subsection{Le flou croissant de la Region en aménagement}

De nombreux géographes allemands ont travaillé en lien avec les organismes de l'aménagement, ce qui fait que les évolutions des concepts et des outils de l'aménagement ont pu avoir des répercussions sur ceux de la géographie et inversement. Dans le cas de la Region, les définitions des ouvrages d'aménagement ont ainsi longtemps mis en avant des caractères d'unicité de type territorial, dans la continuité de l'approche géographique classique de la notion, qu'elle soit région naturelle - E. Meynen (1962) - ou région économique - K.-H. Hottes et alii (1972). De même, M. Sinz (1995) désigne la région comme une " entité spatiale de taille moyenne »- entre l'échelon du Kreis et celui du Land 
- "caractérisée par des spécificités permettant sa reconnaissance et un sentiment d'appartenance». Il esquisse ensuite une typologie différenciant les régions administratives des régions économiques et des régions culturelles, et classe les régions d'aménagement dans les deux premières catégories: elles se fondent sur le regroupement des Kreise (définition administrative) et sur un réseau de villes centrales pertinent (définition fonctionnelle). De fait, comme j'aurai l'occasion d'y revenir ensuite, beaucoup de régions d'aménagement correspondent à l'aire d'attraction d'une ville moyenne ou d'une ville centrale (Oberzentrum) dont elles reprennent le nom. On peut le voir sur la figure l : régions d'aménagement "Aachen », "Stuttgart », " Münster », "Würzburg », «München », etc.

Or, à la fin des années 1990, la Region connaît un renouveau en aménagement et se complexifie. La notion s'élargit et tend à se déterritorialiser en faisant de plus en plus référence à des éléments informels et non territorialisés. Ainsi, en 1995, la conférence ministérielle pour l'aménagement de l'espace décide la mise en place d'un nouvel instrument pour l'action : les «concepts de développement régional ( regionale Entwicklungskonzepte ou $R E K)$. Ils se fondent sur la mise en place de projets coopératifs novateurs dans un cadre dit "régional ", mais situé en fait au sein des régions d'aménagement: on pourrait ainsi associer les $R E K$ au terme français de développement local. C'est alors que la Region commence à être de plus en plus conçue comme un cadre coopératif local et relativement informel, foyer de projets, eux-mêmes coordonnés par l'échelon de la région d'aménagement. Ce «changement des paradigmes de l'aménagement ${ }^{8}$ trouve son inscription législative en 1998, lors de la réforme de la loi sur l'aménagement de l'espace (BauROG: Raumordnungsgesetz). Le paragraphe 13 de la loi indique que les instances de planification au niveau du Land et de la région d'aménagement se doivent d'encourager la coopération entre acteurs publics et privés, en particulier dans le cadre local et contractuel des «concepts de développement régional » $(R E K)$. Ceux-ci sont désignés par le $B B R$ (2000) comme de nouveaux instruments, informels, flexibles et d'initiative locale. Ils reposent sur l'association de partenaires privés (groupes d'intérêts, entreprises) et publics (communes, Kreise) en vue de la mise en œuvre d'actions concrètes : création d'une zone d'activité, développement d'infrastructures de transport régionales, etc. L'échelle retenue est dite "régionale » : il s'agit d'espaces de coopération, situés - sans plus de précision - au-dessus des communes et des Kreise, et au sein de la région d'aménagement. Celle-ci reste l'organe de planification et de coordination de ces projets régionaux.

Cette multiplication des référents régionaux contribue à un réel flou conceptuel. R. Bövermann (2000) explique ainsi que si la région a eu longtemps une définition spatiale (espace d'échelle moyenne, subnationale), elle

8 Expression rencontrée dans la brochure REK in Thüringen, présentant dans une première partie les évolutions des politiques d'aménagement dans le Bund. 
est aujourd'hui devenue un "entrelacs de multiples éléments ». De fait, à côté de la seule région d'aménagement, d'autres entités, ayant un statut juridique plus ou moins établi, sont évoquées en tant que Regionen. Les termes peuvent varier selon les Länder ou selon le type de coopération : " association régionale " (regional Verband), "association de communes" (Kommunalverband), «association de planification» (Planungsverband), etc. Ces termes font référence aux cadres régionaux les plus formels, mais d'autres organisations régionales, faiblement institutionnalisées, sont également encouragées directement, depuis 1998, par les gouvernements fédéral et fédérés (Bövermann, 2000) : les "réseaux de villes", les «conférences régionales ${ }^{9}$, etc. Or, ces entités se fondent beaucoup moins sur des espaces précis que sur des réseaux, en particulier des réseaux d'acteurs.

\subsection{La Region, une entité associative réticulaire}

La coopération et l'association sont devenus les mots d'ordre des politiques actuelles d'aménagement revalorisant l'échelon régional. Or, cette coopération, plutôt que d'être territoriale, comme dans le cas français avec les Pays, est une coopération avant tout sectorielle et thématique. Les projets émergent autour d'un thème (tourisme, transport, emploi, habitat, etc.) qui va impliquer une coordination, une mise en réseau d'acteurs. On pourrait alors parler de projet de réseaux plutôt que de projets de territoires. La cohérence territoriale n'est pas posée, a priori, comme le point de départ d'élaboration des projets locaux. Globalement, ce sont les coopérations les plus souples, et non les coopérations juridiquement instituées, que le $B B R$ encourage le plus : il faut mettre en place «davantage de contrats et de projets au lieu de programmes et de plans », en valorisant ainsi une approche "par le bas ", plus souple et informelle, mais aussi plus proche de la réalité et donc potentiellement plus opérationnelle que par le passé.

Les réseaux de villes (Städtenetze), qui rencontrent un réel succès en Allemagne, sont une belle illustration de cette conception de la région comme entité associative et comme organisation faiblement institutionnalisée qu'encouragent les actuelles politiques d'aménagement. Ils se constituent pour permettre à des villes de renforcer leur poids alors qu'individuellement elles ont du mal à faire face au contexte de concurrence régionale. Cette mise en réseau est par ailleurs encouragée, car elle permet de « renforcer une structure spatiale décentralisée » $(B B R, 2000)$. Elle est fondée sur le volontarisme local, autour de la constitution de projets thématiques: mise en place d'un réseau de transports en commun unifié (ÖNPV), d'un catalogue régional d'offres touristiques, sportives ou culturelles, marketing régional destiné aux entreprises, etc. Chaque réseau de villes accorde une place majeure aux élus

9 Les conférences régionales (ex. Regionalkonferenz Bremen/Niedersachsen, Regionale Entwicklungskonferenz Halle/Leipzig), visent à faciliter la discussion et l'échange entre les acteurs régionaux. Les réseaux de villes, étudiés plus précisément ensuite, sont des coopérations intercommunales, tournées vers la mise en œuvre concrète d'actions régionales. 
(Rat der Bürgermeister) mais dispose de son organisation et de ses objectifs propres. L'idée est de mettre en commun une part des finances de chaque ville membre afin de mener les actions à une échelle régionale, concernant l'ensemble du réseau de villes - un peu sur le modèle intercommunal français, mais dans un cadre plus réticulaire que territorial. En effet, les réseaux de villes sont assez souvent indépendants des maillages territoriaux. Certains passent au-delà des limites des Länder, comme le Sächsisch-Bayerisches Städtenetz (Hof, Bayreuth, Plauen, Chemnitz, Zwickau), voire au-delà des limites nationales : réseau Quadriga entre l'Allemagne et le Luxembourg (Bitburg, Trier, Luxembourg), réseau ANKE entre l'Allemagne et les Pays-Bas (Arnehm, Nijmegen, Kleve, Emmerich). Il existe une grande diversité de tailles de ces réseaux, qui sont considérés comme des régions. Certains ont d'ailleurs fait le choix d'intégrer le terme de Region dans leur nom, comme le réseau EXPORegion, en Basse-Saxe. Le logo de cette région a, par ailleurs, ceci d'intéressant qu'il représente les huit villes membres par des carrés colorés, sans distinction de taille, toutes reliées entre elles par des lignes ouvertes sur l'extérieur, le tout constituant une forme de maille quasi parfaite, qui n'a en fait rien du territoire et tout du réseau... On a ici une belle illustration de ce qui serait une région réticulaire, et qui pourrait constituer le modèle actuel des expériences de développement régional en Allemagne.

\subsection{La constitution de régions réticulaires. L'exemple des réseaux de villes de Thuringe du Sud-Ouest}

Dans la région d'aménagement de Thuringe du Sud-Ouest ${ }^{10}$, trois réseaux de villes ont été l'objet de nos investigations. L'idée était d'interroger les modalités de mise en œuvre de projets régionaux de développement "par le bas » au sein d'un espace régional d'aménagement "par le haut » (région d'aménagement), afin de tenter de mieux appréhender, par une approche concrète de terrain, la notion de «région". L'espace choisi, la région d'aménagement de Thuringe du Sud-Ouest, est à la fois un espace de planification issu d'un découpage exogène, et un territoire présentant une cohérence interne réelle. En termes d'héritages, la région se distingue d'abord du reste de la Thuringe, en ce qu'elle fait partie culturellement et linguistiquement de la Franconie. Par ailleurs, la région d'aménagement reprend quasiment les limites de l'ancien Bezirk ${ }^{11}$ de Suhl qui a été durant plusieurs décennies la référence territoriale et administrative pour les habitants de la région. Sur un plan paysager, comme l'illustre la figure 2 , elle correspond à la vallée de la Werra et au versant sud de la forêt de Thuringe, espace de moyenne montagne (touristique) qui accentue la coupure avec le reste de la Thuringe. La Rhön, région de collines concernant deux autres Länder, est un élément fort d'identification également. Sur un plan fonctionnel, la

10 Il s'agit de l'une des 4 régions d'aménagement/de planification du Land de Thuringe, situé autrefois dans I'ex-RDA, aujourd'hui au centre de I'Allemagne, au nord de la Bavière.

11 Le Bezirk était une circonscription-relais de l'État central, au temps de la RDA. 
région est organisée autour de deux pôles ${ }^{12}$ : Eisenach et Suhl/Zella-Mehlis. Enfin, par sa situation aux confins de l'ex-RDA, à proximité de deux anciens Länder, la Bavière et la Hesse (fig. 2), la Thuringe du Sud-Ouest est aussi un terrain d'analyse particulièrement intéressant en ce qui concerne le poids des effets de frontière sur la mise en œuvre des projets de développement régional, censés les dépasser.

Dans ce contexte, plusieurs réseaux de villes ont fait l'objet de nos analyses, en tant que vecteurs de projets de développement régional. L'association de villes de Thuringe du Sud (Städteverbund Südthüringen) regroupe six villes situées à moins de trente kilomètres les unes des autres. Ce réseau s'est constitué d'abord autour de deux villes (Suhl et Zella-Mehlis) dans le cadre d'un concept de développement régional $(R E K)$. L'objectif était de créer une coopération entre ces deux villes moyennes, en vue de la constitution d'un possible centre urbain de premier ordre ou ville centrale (Oberzentrum ${ }^{13}$ ) pour toute la région d'aménagement. Les deux villes retenues au départ ne rassemblant pas suffisamment de fonctions centrales, le réseau a été élargi à quatre villes moyennes voisines: Schmalkalden, Meiningen, Oberhof et Hildburghausen (fig. 3). L'ensemble des six villes pourrait ainsi théoriquement, d'après une étude réalisée par le Pr. Dr. Usbeck (Leipzig), prétendre au rang d'Oberzentrum, auquel seules Jena, Erfurt et Gera ont pour l'instant accédé en Thuringe.

En fait, l'approche du Städteverbund est très audacieuse : il s'agit de faire passer l'idée non pas d'une ville centrale, mais d'un réseau de villes central, d'une région (Region) centrale fondée sur un réseau. Or, le Land et la région d'aménagement ne conçoivent un Oberzentrum que sous une forme ponctuelle, limitée spatialement à une ville.

Il se dégage ainsi une réelle différence de perception, source de conflit, entre la "région » en construction (celle du réseau de villes) et la région d'aménagement, fidèle à une vision classique plus territorialisée. Le Städteverbund n'a pas encore été reconnu comme Oberzentrum mais ses acteurs (les maires et les représentants des Landkreise) travaillent de plus en plus étroitement, désormais autour d'un manager régional, pour tenter de le faire reconnaître lors du prochain plan de développement spatial du Land. Ils valorisent ainsi - auprès de la région d'aménagement et de la population - les actions illustrant la réussite de la coopération actuelle : réseau régional de transport en commun (ÖNPV), construction d'une zone industrielle de 400 ha, création d'une carte d'accès aux principaux sites touristiques, mise en place d'une base de données commune sur les hébergements touristiques des six villes, etc. Le Städteverbund espère mobiliser ces actions comme autant

12 Il s'agit de deux villes moyennes aux fonctions partielles de villes centrales. Chaque région d'aménagement est ainsi organisée autour d'une ou plusieurs villes de rang supérieur, de même que chaque Kreis est organisé autour d'une ou plusieurs villes de rang inférieur.

13 La théorie des places centrales est une grille majeure de l'aménagement de l'espace en Allemagne. L'intérêt d'être reconnu "Oberzentrum» par la région de planification puis le Land réside dans l'attractivité qui est alors associée à ce titre de ville centrale. 


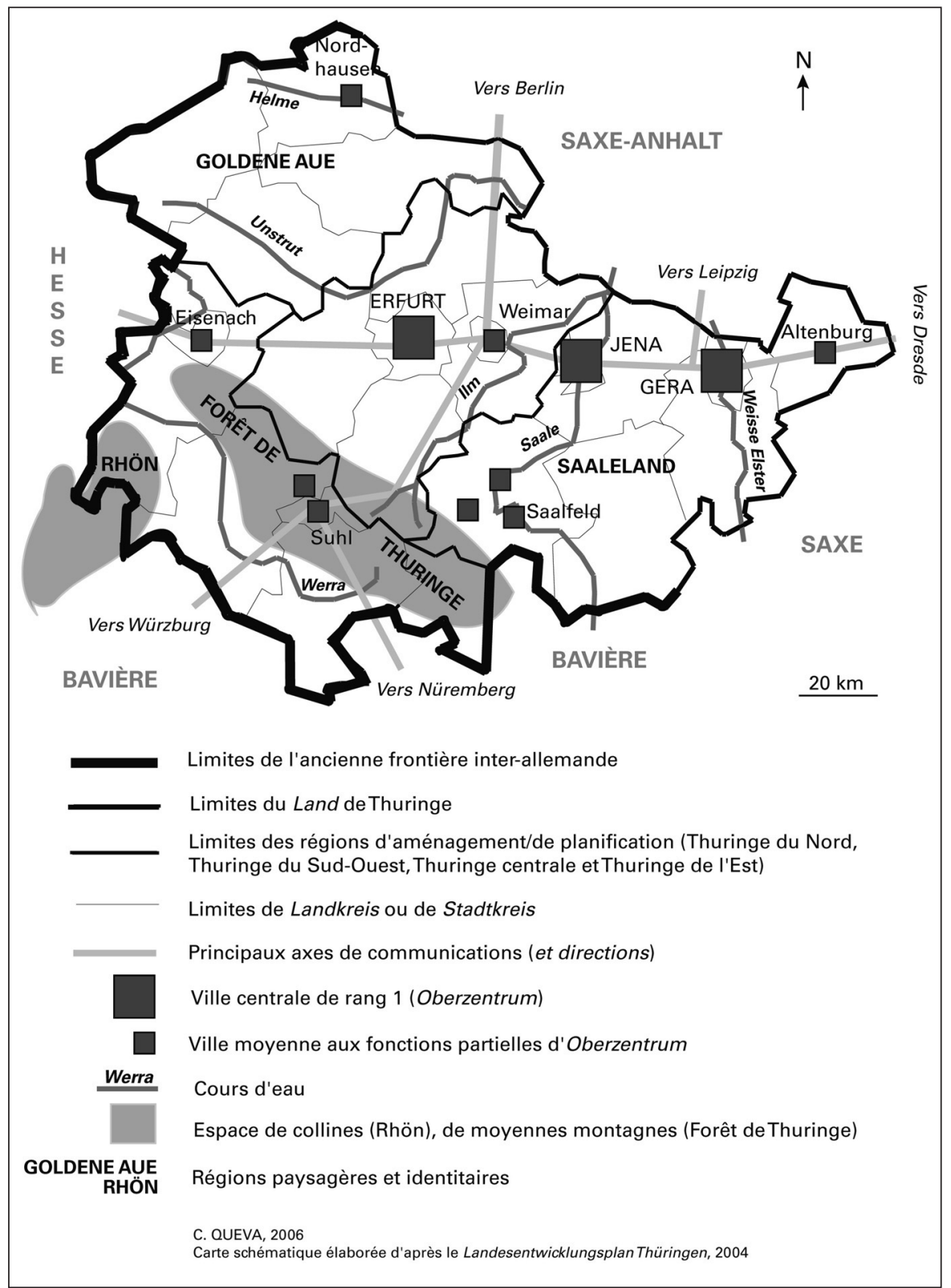

Fig. 2 Régions d'aménagement et cadre géographique en Thuringe. Spatial development regions and Thuringe geographic setting. 


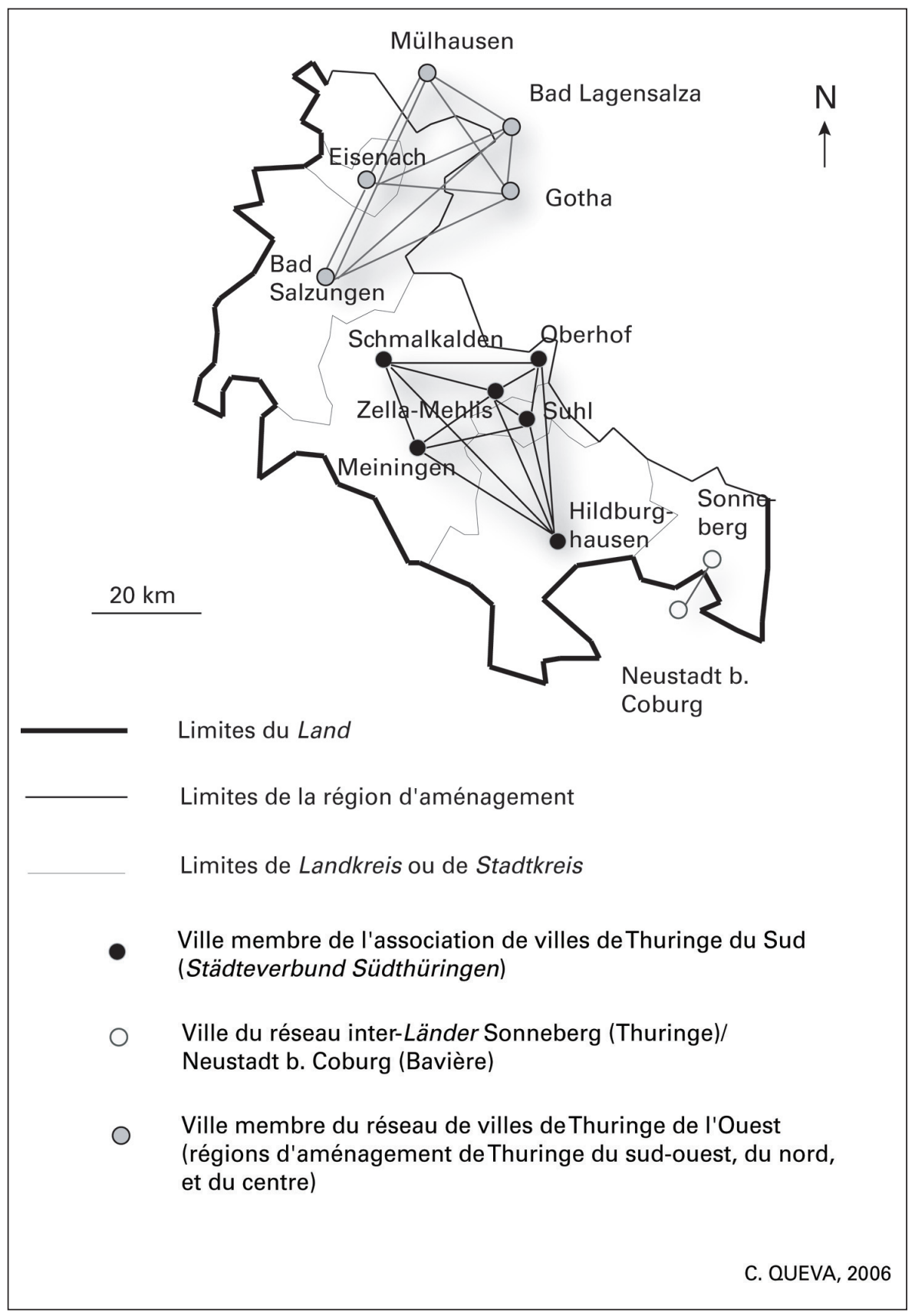

Fig. 3 Les réseaux de villes en Thuringe du Sud Ouest. Urban network in South-West Thuringe. 
d'arguments pour sa reconnaissance par la région d'aménagement et le Land en tant que réseau de villes central (Oberzentrum), l'objectif majeur qui n'a toujours pas été atteint.

Sur ce plan, le relatif échec du Städteverbund Südthüringen s'explique également par la vive concurrence qui s'est établie avec les autres villes de la région d'aménagement, notamment Eisenach et Sonneberg qui, chacune de leur côté, ont tenté également de constituer un réseau de villes (fig. 3). Le réseau de villes organisé autour d'Eisenach, en Thuringe de l'Ouest (kommunale Arbeitsgemeinschaft Westthüringen), s'est clairement mis en place pour contrecarrer et faire échouer la reconnaissance du Städteverbund Südthüringen en tant qu'Oberzentrum, comme nous l'a expliqué le maire d'Eisenach, ville moyenne dynamique. L'idée d'un réseau de villes central autour de Suhl, qui placerait Eisenach sous sa tutelle et en réduirait ainsi l'influence était inimaginable. Le réseau de villes de Thuringe de l'Ouest s'est alors constitué au même moment et avec la même intention que le Städteverbund Südthüringen: devenir un Oberzentrum. L'objectif a été atteint : le Land ne pouvait pas reconnaitre trop de villes centrales dans son plan de développement spatial, et il n'en a donc reconnue aucune. On retrouve ici le conflit, évoqué plus haut, entre la région d'aménagement et les «régions» (réseaux de villes) qui la composent. Ce conflit est paradoxal, dans le sens où les "régions" ne peuvent être reconnues par le Land - et donc recevoir des subventions plus importantes - que par l'intermédiaire de la région d'aménagement ${ }^{14}$. Le dernier réseau de villes étudié est un projet de coopération par-delà les frontières entre les Länder (fig. 3) et par-delà les disparités économiques entre la Thuringe (Sonneberg) et la Bavière (Neustadt b. Coburg). L'objectif était de recréer le lien qui existait autrefois entre les deux villes, afin de constituer une région économique (Region) unie et viable. Il fonctionne en fait au ralenti, les différences de juridiction et de fonctionnement entre les deux Länder ainsi que leur différentiel économique étant les principaux obstacles à la coopération.

Ces trois exemples sont une illustration du changement des paradigmes de l'aménagement. L'émergence des projets et des coopérations se fait "par le bas », dans un cadre régional qui se crée par le réseau. Dès lors, la Region n'est pas un donné, elle se construit : en tant qu'espace de développement et d'action, elle se caractérise comme une entité plus associative que territoriale, au caractère plus souple qu'institutionnel. Elle est marquée par un souci de détachement vis-à-vis des structures qui constituent son cadre (Kreise, Länder, régions de planification). Mais comment expliquer cette volonté de se détacher à ce point des structures fermes, et en particulier des structures territoriales ? Il semble que la réponse soit à rechercher

14 Les régions des réseaux de villes s'inscrivent également dans le cadre planifié et emboîté évoqué précédemment (Landesplanung, Regionalplanung, etc.). Ce cadre, s'il est censé évoluer vers "davantage de contrats et de projets au lieu de programmes et de plans » $(B B R)$, n'en reste donc pas moins parfois un frein à ces projets. 
dans une certaine conception de l'espace, rencontrée notamment au cours des enquêtes effectuées dans la région d'aménagement de Thuringe du Sud-Ouest ${ }^{15}$ : le territoire y a été présenté non pas comme le point de départ (central) mais plus comme un éventuel point d'aboutissement (secondaire) des expériences de développement régional.

\section{La Region, entre déterritorialisation par le projet et reterritorialisation par I'action}

Le rejet des approches territoriales peut être justifié, à l'est de l'Allemagne, par l'héritage socialiste. Le territoire était alors au cœur des approches planifiées de l'aménagement, dans le cadre de la planification territoriale (Territorialplanung), effectuée à plusieurs niveaux emboîtés, dont le territoire de la RDA et celui des Bezirke, circonscriptions administratives appliquant les directives de l'État central. Plus généralement, le terme Territorium (territoire) conserve, en Allemagne, cette connotation hiérarchique, ferme et directive. Il apparaît donc quasiment en contradiction avec les politiques de développement endogène actuelles.

\subsection{Un double détachement vis-à-vis de l'espace et des institutions : réseaux contre territoires?}

Dans la discipline géographique allemande, la région connaît, depuis les années 1980, un renouveau réel, cette Nouvelle Géographie Régionale délaissant les perspectives classique - la région traditionnelle comme unité spatiale ou paysagère - et analytique - la région comme catégorie d'analyse - au profit d'une approche plus sociale, centrée sur les pratiques et les représentations: celle de la région comme construction individuelle et subjective (Miggelbrink, 2002). La nature de la région réside ainsi non plus dans ses caractères en tant que tels, mais dans ceux que lui attribuent un individu ou un groupe social (B. Werlen, 1997). Par extension, l'espace (régional) en tant que tel compte peu; il n'est que le résultat des actions, des représentations et des objectifs de la société qui le vit et qui le pratique. La région est ainsi de plus en plus conçue comme un espace d'action (Knieling, 2003). Par opposition au territoire, hiérarchique, délimité, associé aux approches exogènes, la région est l'espace du développement endogène, des acteurs locaux qui lui donnent ainsi tout son sens. "Dans le contexte de la science et de l'aménagement, la notion de région est comprise comme une abstraction spatiale d'échelle moyenne ayant un (ou des) objectif(s), et dont la dimension spatiale et les limites se déduisent de l'(ou des) objectif(s) qui lui ont été attribués»(Wollf, 2002). La région tend donc, dans la géographie sociale allemande actuelle, à être conçue comme une construction sociale, un tissu de relations entre les acteurs régionaux : elle se définit alors davantage par l'intensité de ces relations que par une surface

1524 entretiens effectués auprès des principaux acteurs du développement régional. 
délimitée ou un territoire. C'est ce qu'évoquent E. Bergmann et U. Hardt (1999), reprenant les propos de H. Mäding ${ }^{16}$ : «les régions sont traditionnellement définies de manière territoriale (territorial definiert), en tant qu'unité spatiale présentant des propriétés spécifiques; toutefois, il se développe aujourd'hui une conception basée sur la coopération, qui est moins marquée par l'image de la surface (Fläche) que par celle du réseau d'acteurs (Akteursnetzes) ».

En aménagement, ce détachement vis-à-vis de l'espace se manifeste par une certaine méfiance vis-à-vis de l'institutionnel. On a déjà pu mentionner le rejet, dans les textes actuels de l'aménagement, des organisations formelles trop figées au profit d'expériences coopératives relativement informelles. À l'opposé de celles-ci, les approches territoriales incarnent l'institutionnel, la hiérarchie et la fixité. Dans ce cadre, on comprend que les nouvelles politiques d'aménagement - souhaitant le renforcement des structures régionales souples et informelles pour laisser émerger les innovations et les projets porteurs - tendent à encourager les réseaux au détriment du territoire. Ce détachement vis-à-vis du territoire pourrait ainsi s'inscrire dans cette spécificité allemande, marquée, selon D. Fürst (2001), par un réflexe de «fuite hors des institutions ». Le développement local se fonde donc en Allemagne avant tout sur une approche réticulaire et non territoriale de l'action. Les deux démarches sont généralement posées comme antithétiques : l'approche territoriale correspond à une démarche de planification et d'aménagement "par le haut» (Ansätze von oben), alors que c'est dans des réseaux informels de coopération que doivent émerger les projets de développement, élaborés par les acteurs locaux (Initiative von unten). B. Müller (1999) oppose ainsi la coordination spatiale et la coopération régionale: « räumliche Koordination versus regionale Kooperation». Ce cadre coopératif repose sur l'idée d'initiative, dans un contexte concurrentiel (Wettbewerbe) que le $B B R$ souhaite encourager, sans le formaliser: les projets restent le fait d'acteurs locaux qui, en se regroupant, forment une Region, volontairement flexible, associée simplement à l'idée de coopération, non limitée spatialement : "ce n'est que dans les cas d'exception que des unités spatiales strictes sont proposées", comme l'explique la brochure $R E K$ in Thüringen.

\subsection{La gouvernance régionale, un contre-poids aux pouvoirs territorialisés}

Dans ce double contexte de détachement vis-à-vis du territoire et des institutions, et de renforcement de la région comme entité coopérative et réticulaire, se développe de plus en plus un autre concept: la gouvernance régionale (regionale Governance). La notion de gouvernance est assez difficile à définir. Elle désigne une forme de coordination sociale et d'action collective, à côté du système de pouvoir organisé. D. Fürst (2001) reprend

16 H. Mäding, "Perspektiven für einer Europa der Regionen », in Aktuelle Informationen des Deutschen Instituts für Urbanistik, Berlin, Dezember 1998, p. 4. 
dans son article la définition qu'en a donné la Commission sur la gouvernance mondiale en $1995^{17}$. On pourrait la résumer en disant que la gouvernance, action collective coopérative, s'appuie sur une pluralité de modalités d'actions, marquée par l'association d'individus, d'institutions publiques et/ou d'acteurs privés. Elle se base donc sur des instances formelles et informelles, et sur des structures faiblement institutionnalisées telles que les réseaux, les tables rondes, etc. À cet égard, elle se différencie du gouvernement (goverment), terme désignant le système politique institutionnalisé (institutionalisierte Steuerungssytem). La gouvernance est, quant à elle, un système de régulation (Regulierungssystem).

C'est en ce sens qu'en Allemagne, le terme de regionale Governance (gouvernance régionale) a pu se forger : comme nous l'avons vu, la région est un cadre coopératif, relativement informel et réticulaire. Elle est donc présentée par D. FÜRST comme le contexte idéal pour la mise en place d'un système d'action collective, ayant une organisation autonome, dans laquelle tous les acteurs sont interdépendants. Ce nouveau paradigme de l'action trouve alors son incarnation dans les expériences régionales telles que les réseaux de villes, certains ayant pour objectif de créer un contre-pouvoir face aux territoires traditionnels. Le cas du Städteverbund Südthüringen, évoqué plus haut, en est un bel exemple. En souhaitant créer un Oberzentrum sous la forme d'un réseau, les maires des six villes membres, regroupés dans un conseil (Rat der Bürgermeister), cherchent aussi à proposer une nouvelle forme de gestion et d'organisation des pouvoirs : ils s'efforcent d'intégrer les réflexions de groupes de travail thématiques (tourisme, économie, infrastructures, etc.) regroupant partenaires privés et publics, que ce soit les Lankreise, les entreprises, les associations, les chambres consulaires, etc. On est bien là dans une perspective de gouvernance régionale. C'est alors au manager régional que revient la tâche de stimuler et d'entretenir cette gouvernance - autrement dit les liens entre les différents acteurs de la région formée par le Städteverbund. Un autre exemple pourrait être pris avec la Rhön, région de moyenne montagne et réserve de biosphère, située au sud-ouest de la région et à cheval sur trois Länder: la Thuringe, la Bavière et la Hesse. La gestion de cet espace est partagée entre les six Landkreise concernés, le groupe de travail central étant composé des Landräte (élus des Lankreise). Mais, là encore, différents groupes de travail (portail internet, identité, tourisme) essaient de mobiliser les acteurs régionaux, qu'ils soient du domaine public ou du domaine privé. Enfin, un management régional a également été mis en place dans cette région.

La Region, d'après les politiques actuelles d'aménagement et les approches des scientifiques, notamment des géographes, est donc un cadre d'action et de coopération réticulaire avant d'être territorial, le territoire étant associé à l'idée de cadre exogène et hiérarchique. La réussite des projets d'aménagement et de développement résulterait alors de la capacité des

17 Commission on Global Governance, Nachbarn in einer Welt. Der Bericht der Kommission für Weltordnungspolitik, Bonn, 1995. 
acteurs à se doter de structures régionales suffisamment souples et ouvertes pour ne pas trop se territorialiser. C'est ainsi que s'est établi le flou conceptuel associé à la notion de région, évoqué plus haut. La coopération régionale peut même en venir à être considérée comme synonyme d'intercommunale : "Vielfach werden die Begriffe "interkommunale" und "regionale" Kooperation synonym verwandt» (Danielzyk, 1999). Cette souplesse et ce flou du concept régional n'en restent cependant pas moins l'objet de critiques, notamment quant à l'articulation des jeux d'acteurs endogènes et exogènes, et des échelles supra- et infra-régionales, et donc à la nécessaire revalorisation d'un échelon régional territorialisé.

\subsection{Le flou régional en question}

L' « inflation de coopération régionale » (Diller, 2002) aboutit à environ 400 cas de coopérations, alors que les régions d'aménagement ne représentent que 97 entités en Allemagne. Ce contraste entre le Wo (où) et le Wie (comment) est, selon C. Diller, un signe d'imperfection et de difficulté dans la coordination des projets. Il y a donc une nécessité à articuler ce " où » et ce " comment », le spatial et le réticulaire, au lieu de chercher nécessairement à les isoler comme dans les actuelles pratiques de coopération et de planification. La région d'aménagement de Thuringe du Sud-Ouest est, à cet égard, assez représentative. Elle connaît, comme beaucoup d'autres en Allemagne, une multiplication des expériences réticulaires plus ou moins informelles, ce qui aboutit à un enchevêtrement de ces réseaux, et à une confusion terminologique réelle : à quoi pense-t-on quand on parle de «région »? Les réponses sont multiples. La région d'aménagement/de planification (Thuringe du Sud-Ouest) est la région-cadre, dans laquelle s'établissent les projets régionaux (Regionen). Ceux-ci, en tant que mises en réseaux d'acteurs locaux, constituent des régions de coopération, telles que celles formées par les réseaux de villes (fig. 3). À cela s'ajoutent les deux espaces de collines et de moyennes montagnes, la Forêt de Thuringe et la Rhön (fig. 2). Il s'agit de deux régions touristiques, à l'identité marquée pour les populations locales, mais aussi de deux régions de coopération; on l'a vu avec la région Rhön, dont la gestion est partagée entre les acteurs de trois Länder. Pour la forêt de Thuringe, une "régionalisation » est actuellement en cours, dans le sens où l'on tente de regrouper sous le label Forêt de Thuringe et dans une seule association régionale (Regionalverbund Thüringer Wald), créée en septembre 2004, tous les acteurs du tourisme travaillant sur cet espace "naturel », qui s'étend sur trois régions d'aménagement (de Thuringe du Sud, du Centre et de l'Est). Dans tous les cas, ces Regionen sont en construction et résultent d'initiatives locales. Elles correspondent à des projets de développement régional, effectués par des coopérations souvent associatives. Mais elles restent des coopérations ouvertes, en cours, et sans fondements administratifs et territoriaux fermes.

Or, c'est peut-être justement un ancrage territorial et institutionnel qui manque à ces projets, afin de les rendre plus porteurs et plus durables. D'ailleurs, la référence à une meilleure coordination de ces projets, trop 
éclatés, a souvent été évoquée dans les entretiens. En parallèle à une territorialisation des projets locaux (Regionen), la région d'aménagement aurait alors à accentuer les approches en termes de réseaux pour améliorer cette coordination des initiatives locales. La difficulté essentielle réside en fait dans la relative faiblesse de la région d'aménagement, faiblesse reconnue par le BBR (1999), et expliquée notamment par l'éclatement des niveaux d'action pour les espaces d'échelle moyenne (ministères du Land, associations intercommunales et régionales, gouvernements des Kreise, associations informelles, etc.). Le choix offert aux acteurs d'établir leurs projets dans le cadre de structures souples et ouvertes, s'il est un bien dans la formulation et la constitution de ces projets, devrait donc ensuite, aux dires des aménageurs et des personnes enquêtées, déboucher sur des structures plus fermes, aux contours juridiques et spatiaux plus clairement définis. La région d'aménagement pourrait ainsi constituer un échelon d'action (et plus seulement de planification) particulièrement pertinent, en tant que territoire fonctionnel.

\subsection{La région d'aménagement comme espace pertinent}

Le souci de fonctionnalité et de structure de l'espace de la région d'aménagement tend, par ailleurs, à être de plus en plus mis en évidence. Créées en 1981 pour appliquer les politiques de planification des Länder et revalorisées en 1998 en tant qu'échelon de coordination des projets régionaux réticulaires, les régions d'aménagement ont commencé, dès 1982, à être conçues comme des espaces fonctionnels, tout en restant basées sur les circonscriptions administratives des Kreise. Elles sont ainsi organisées autour d'une ville centrale de rang intermédiaire ou supérieur (Mittelzentrum ou Oberzentrum), de même que les Kreise ont des systèmes de lieux centraux de rang inférieur. C'est ainsi que M. Sinz (1995) évoque la nécessité de concevoir les régions d'aménagement comme des entités mixtes, à la fois homogènes et fonctionnelles, pour reprendre la terminologie qu'il utilise. Les régions homogènes, basées sur le principe de similitude, sont des unités naturelles, paysagères, linguistiques, ou encore de conscience régionale. Les régions fonctionnelles se fondent, quant à elles, sur des relations d'interdépendance, qui ne sont pas toujours clairement identifiées. Il s'agit, par exemple des bassins d'emplois ou encore des espaces marqués par une interdépendance de lieux centraux. Or, pour M. Sinz, et même si cette perspective peut sembler de l'ordre de l'utopie, les régions d'aménagement constituent un type original, mixte (Mischtyp), marqué par la conjonction de ces deux catégories : elles ont une homogénéité structurelle qui doit être conçue comme un point de départ pour la formation d'une région fonctionnelle. Cette fonctionnalité spatiale des régions d'aménagement est alors, d'après M. Sinz, à valoriser et à accentuer dans le cadre de coopérations intercommunales et de réseaux de villes, mais sous une forme juridique stable, et non plus seulement de manière informelle. Il serait ainsi de plus en plus souhaitable que la région d'aménagement, pour son bon fonc- 
tionnement et pour une plus grande efficacité de l'action, s'appuie davantage sur les structures administratives et territoriales existantes : de l'articulation des territoires institutionnels et fonctionnels semble aujourd'hui dépendre l'avenir des régions d'aménagement.

Enfin, dans une perspective plus identitaire, de nombreux projets sont en cours afin d'ancrer territorialement, sur les plans matériel et symbolique, les régions d'aménagement. Chacune est désormais dotée de son site Web, où se trouvent généralement en ligne non seulement les projets de planification régionale (en tant que relais du Land) et les projets coopératifs régionaux mis en place par les acteurs locaux, mais aussi tout un discours sur la fonctionnalité, voire l'identité de la région. Sur un plan fonctionnel, les sites insistent généralement sur les lieux centraux qui font l'unité de la région. Sur un plan symbolique, les régions d'aménagement sont toutes dotées d'un logo représentatif ayant souvent beaucoup d'aspects territoriaux, par la représentation directe ou symbolique (carrefour autoroutier, montagne, rivière, etc.) du territoire de la région d'aménagement.

\subsection{Des constructions régionales en cours : sur le chemin de la territorialisation des Regionen}

En parallèle à cette territorialisation des régions d'aménagement, les espaces locaux eux-mêmes (Regionen), pour l'instant systèmes de coopérations régionales informelles, semblent également susceptibles de connaître un paradoxal retour à l'institutionnel, aux structures fermes et au territorial. Cette dynamique s'observe dans le cas des régions de Thuringe du Sud Ouest. En effet, si chacune d'entre elles repose sur une coopération entre acteurs privés et publics, sur des réseaux (de villes, d'acteurs) et sur des structures souples de type associatif, autour d'un manager régional (gouvernance), elles ne doivent leur réussite et leur persistance qu'à un seul acteur, territorial : le Landkreis (arrondissement). Cet échelon administratif local, situé au-dessus des communes, a à sa tête un conseil élu, le Kreistag, et une personnalité politique, le Landrat. Il s'agit d'une structure territoriale classique de gouvernement. Or, elle a été présentée dans les entretiens comme l'acteur et l'échelon de référence pour tout projet de développement régional, en tant qu'organe de financement, de coordination et de lisibilité des projets vis-à-vis des échelons supérieurs, et notamment de la région d'aménagement. Autrement dit, toute gouvernance reposerait en fait sur une structure classique de gouvernement territorialisé... C'est ainsi, par exemple, que le Städteverbund Südthüringen, tout informel et ouvert qu'il soit (par exemple vers la ville universitaire voisine d'Ilmenau), implique en fait la nécessaire prise en compte d'un territoire formé de deux Landkreise (Schmalkalden-Meiningen et Hildburghausen), et de la ville-Kreis de Suhl. La région que représente "réellement» le Städteverbund n'est alors pas seulement la région réticulaire théorique, relativement floue, des six villes membres, mais aussi une région territoriale et administrative, strictement délimitée (fig. 4). C’est dans ce cadre territorial qu'évolue le Städteverbund: 
toute commune membre de ce territoire est concernée par ses projets et ses actions. En termes de jeux d'acteurs, même si ce sont les maires qui restent à la tête du Städteverbund, une personnalité politique domine largement. Il s'agit du Landrat Luther (Landkreis de Schmalkalden-Meiningen). En effet, dans la mise en place de la dynamique de réseau de villes, c'est lui qui a amené les maires à se réunir et à réfléchir à un projet de coopération, et qui reste le premier nom cité associé au développement régional du Städteverbund. Ce sont donc le Landkreis et le Landrat qui, aux côtés des maires, sont les acteurs centraux du développement régional. Il en est de même dans la région Rhön où les Landkreise sont les acteurs décisionnels. Le Landrat Luther, initiateur du Städteverbund, est, par ailleurs, président du Forum régional de la région Rhön.

À plus petite échelle, l'association régionale qui se met actuellement en place à l'échelle de la forêt de Thuringe (Regionalverbund Thüringer Wald), cherche, certes, à regrouper les associations touristiques existantes «sous un même toit » souple et associatif - avec à sa tête un manager chargé de valoriser, par un marketing dit "régional ", un espace a priori non administratif transcendant les découpages traditionnels, celui de la forêt de Thuringe. Mais cet espace d'actions et de projets correspond en fait là encore, en termes de jeux d'acteurs, à un territoire bien précis : celui de huit Landkreise et de deux villes-Kreis (fig. 4). L'association a, par ailleurs son président, et il s'agit du Landrat Luther.

La figure 4 représente les régions territoriales les plus manifestes dans l'espace étudié. Chacune est fondée sur des regroupements de Kreise: la région d'aménagement, le territoire « réel » du Städteverbund Südthüringen et celui du Regionalverbund Thüringer Wald.

La terminologie illustre bien l'idée d'une phase constructive, d'une régionalisation en cours. Le terme Südthüringen (Thuringe du Sud) fait ainsi référence à des espaces de tailles diverses allant du territoire du réseau de villes (2 Landkreise) au district de la Chambre de commerce et d'industrie ( 5 Landkreise) ${ }^{18}$, en passant par l'échelon de la région d'aménagement elle-même qui, jusqu'en 2001, s’intitulait également Südthüringen avant de devenir Südwestthüringen - par souci de différenciation avec le Städteverbund... Régionalisation en cours, donc, qui semble être passée par le réseau et les structures informelles dans sa phase initiale (concepts, projets), avant de recourir au territoire dans la phase de l'action. Cette territorialisation s'explique essentiellement par le jeu des acteurs, lui-même marqué par une double dynamique d'ouverture et de gouvernance dans la phase projective, et de repli sur les modes traditionnels de gouvernement par la suite. Il n'en reste pas moins que la coopération entre les acteurs de ces territoires traditionnels des Landkreise reste une nécessité pour le bon fonctionnement des projets régionaux.

18 Industrie- und Handelskammer Südthüringen. 


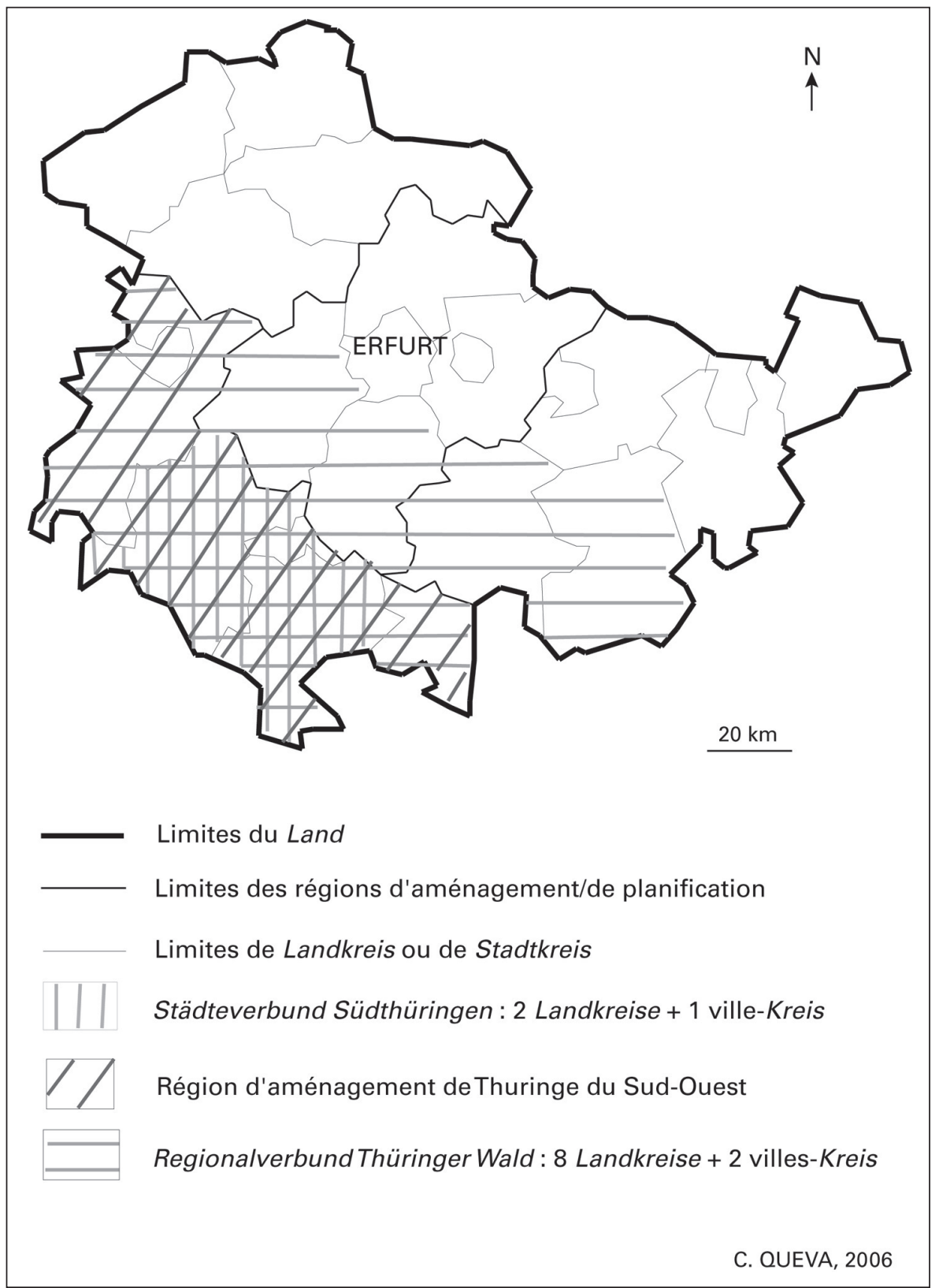

Fig. 4 Exemple de 3 régions territoriales en Thuringe du Sud Ouest.

The case on three territorial regions within South-West Thuringe. 


\title{
Conclusion
}

La notion de région, en Allemagne, a été présentée comme duale, voire plurielle. Dans sa définition en tant que région d'aménagement, elle est conçue comme une entité ancrée et limitée spatialement, censée être fonctionnelle et homogène. Toutefois, au sens large, la Region est plutôt conçue, au regard des expériences de développement régional, comme une entité réticulaire non territoriale - ou tout au moins non revendiquée comme telle - fondée avant tout sur les réseaux coopératifs, d'acteurs ou de villes. En fait, il a été montré que la conjonction des deux approches territoriales et réticulaires, fondée sur l'articulation des jeux d'acteurs et des jeux d'échelles spatiales, pourrait aujourd'hui devenir une nécessité pour mener à bien les projets d'aménagement et de développement régional. La réalité du terrain étudié, les exemples de régionalisations en cours dans le reste de l'Allemagne, rencontrés au fil des lectures, ainsi que la confrontation des expériences régionales avec les projets de territoires que sont les Pays en France, nous laissent penser que la territorialisation des réseaux régionaux pourrait être une condition de leur durabilité et de leur efficacité. La question d'une nouvelle réforme territoriale (Gebietsreform), à l'échelon des Kreise en particulier, est ainsi le souhait le plus vif des acteurs locaux rencontrés : la coopération et la mise en réseau seront d'autant plus efficaces que les structures administratives seront élargies et simplifiées. Dès lors, entre multiplication des approches réticulaires dans le cadre de la région d'aménagement et territorialisation des expériences de coopération régionale, l'Allemagne présente des expériences originales en termes de développement local, susceptibles de constituer des pistes de réflexion intéressantes pour les aménageurs et les acteurs locaux des pays voisins.

\author{
Université Michel de Montaigne (Bordeaux III) \\ Laboratoire ADES (UMR 5185) \\ 12, Esplanade des Antilles \\ 33607 Pessac \\ chrisqueva@hotmail.com
}

\section{Bibliographie}

Baitsch C., Müller B. (2001), Moderation in regionalen Netzwerke, Münschen, Rainer Hampp Verlag, $233 \mathrm{p}$.

Baudelle G., Kunzmann K.R. (2004), « Regards croisés sur l'aménagement du territoire en France et en Allemagne », ESO, travaux et documents, $n^{\circ} 22$, p. 69-77.

Behar D. (2000), "Les nouveaux territoires de l'action publique », in D. Pages, N. Pelissier (dir.), Territoires sous influence, t. 1, p. 83-101.

Bundesamt für Bauwesen und Raumordnung, 1999, « Perspektiven für die Region als Planungs- und Handlungsebene", Informationen zur Raumentwicklung, Heft 9/10, p. 577-712. 
Bundesamt für Bauwesen und Raumordnung, (2000), Raumentwicklung und Raumordnung in Deutschland, Teil 2 : "Raumordnung », p. 43-60.

Bundesamt für Bauwesen und Raumordnung (2001), "Regionen im Wettbewerb », Informationen zur Raumentwicklung, Heft 8, p. 465-516.

Bergmann, E., Hardt U. (1999), "Aufgabenverteilung und Einnahmekompetenzen in Regionen », Informationen zur Raumentwicklung, Heft 9/10, p. 629-644

Bövermann R. (2000), "Regionen », in U. Andersen et alii, Handwörterbuch des politischen Systems der Bundersrepublik Deutschland, Opladen, p. 513-516.

Danielzyk R. (1998), Zur Neuorientierung der Regionalforschung, Oldenburg, BIS-Verlag, 519 p.

Danielzyk R. (1999), "Regionale Kooperationsformen », in BBR, Informationen zur Raumentwicklung, Heft 9-10, p. 577-586.

Diller C. (2000), «Zwischen Netzwerk und Organisation. Die Dynamik der Versteigerung regionaler Kooperationen », Raumforschung und Raumordnung, p. 146-154.

Felgenhauer T., Mihm M., Schlottmann A. (2003), «Langage, Médias, et régionalisation symbolique : La fabrication de la Mitteldeutchland", Géographie et culture, n 47, p. 85-102.

Fürst D. (2001), « Regional governance - ein neues Paradigma der Regionalwissenschaften ? , Raumforschung und Raumordnung, Heft 5-6, p. 370-380.

Fürst D., Schubert H. (2001), «Regionale Akteursnetzwerke zwischen Bindungen und Optionen. Über die informelle Infrastruktur des Handlungssystems bei der Selbstorganisation von Regionen », Geographische Zeitschrift, Heft 1, p. 32-51.

Guder U. (2003), L'aménagement du territoire et la politique régionale en Allemagne: vers une européanisation en douceur?, Groupement d'Études et de recherches « Notre Europe », 47 p.

Gutersohn H., Troll C. (1963), Geographie und Entwicklungsplanung, Basel, Pharos-Verlag, 52 p.

Hellmer F., Friese C., Kollros H., Krumbein W. (1999), Mythos Netzwerke. Regionale Innovationsprozesse zwischen Kontinuität und Wandel, Berlin, Sigma, 288 p.

Hottes K.-H., Meynen E., Otremba E. (1972), Wirtschafträumliche Gliederung der Bundesrepublik Deutschland: geographisch-landeskundlische Bestandsaufnahme, Bonn, Bundesforschungsanstalt für Landeskunde und Raumordnung, 269 p.

Knieling J., Fürst D., Danielzyk R. (2003), Kooperative Handlungsformen in der Regionalplanung. Zur Praxis der Regionalplanung in Deutschland, Dortmund, Dortmunder Vertrieb für Bau- und Planungsliteratur, $221 \mathrm{p}$.

Lacquement G. (2004), "Politique d'intégration territoriale et ancienne frontière inter-allemande », Annales de géographie, n 636, p. 168-187.

Le Gloannec A.-M. (2001), L'État en Allemagne. La République fédérale après la réunification, 458 p.

Marcou G., Kistenmacher H., Clev H.-G. (1994), L'aménagement du territoire en France et en Allemagne, DATAR, La Documentation française, 290 p.

Meynen E. (1962), Handbuch der natürräumlichen Gliederung Deutschlands, Bad Godesberg, Bundesanstalt für Landeskunde und Raumforschung, 8 vol., 1139 p.

Miosga M. (1999), Europäische Regionalpolitik in Grenzregionen : die Umsetzung der INTERREGInitiative am Beispiel des nordrhein-westfälisch-niederländischen Grenzraums, Passau, LIS, $194 \mathrm{p}$.

Miggelbrink J. (2002), Der gezähmte Blick. Zum Wandel des Diskurses über «Raum» und "Region" in humangeographischen Forschungsansätzen des ausgehenden 20. Jahrhunderts, Beiträge zur regionalen Geographie, Leipzig, Institut für Länderkunde, 212 p.

Müller B. (2003), « Regionalentwicklung unter Schrumpfungsbedingungen. Herausforderung für die Raumplanung in Deutschland ", Raumforschung und Raumordnung, Heft 1-2, p. 28-42.

Sinz M. (1995), « Region », in Akademie für Raumforschung und Landesplanung, Handwörterbuch der Raumordnung, VSB, Braunschweig, p. 805-808.

Werlen B. (1987), Gesellschaft, Handlung und Raum : Grundlagen Handlungstheoretischer Sozialgeographie, Stuttgart, F. Steiner, 315 p. 
Werlen B. (1997a), Sozialgeographie alltäglicher Regionalisierungen, Band 1 « Zur Ontologie von Gesellschaft und Raum », Stuttgart, F. Steiner, 262 p.

Werlen B. (1997b), Sozialgeographie alltäglicher Regionalisierungen, Band 2 "Globalisierung, Region, Regionalisierung », Stuttgart, F. Steiner, 464 p.

Wolf K. (2002), " Region », in E. Brunotte et alii (Hrsg.), Lexikon der Geographie, 3, Berlin, Spektrum Akademischer Verlag Heidelberg, p. 126.

Wollmann H. (1996), "The transformation of Local Government in East Germany : Between Imposed and Innovative Institutionalization", in A. Benz, K. Goetz (éd.), A new German Public Sector, p. 137-163. 